\title{
Work In Progress: Utilizing Guided Worksheets to Address Gender Gap in Troubleshooting Laboratory Course
}

\section{Sabia Zehra Abidi, Rice University}

Abidi has a doctorate in biomedical engineering from the University of Texas, Austin. Her investigations in Professor Krishnendu Roy's lab utilized in vitro 3-D polymer scaffolds and notch ligand functionalized microbeads to scale up the production of cells of hematopoietic lineage. Optimization of scaffold and microbead properties resulted in enhanced commitment to hematopoiesis and T cell lineage, respectively, demonstrating promise for cell substitutes in diseases of immunological origin. Abidi also completed postdoctoral research at NYU School of Medicine utilizing microbiological techniques to characterize a unique Plasmodium phenotype - a triggering of parasite death at high densities. The insights have implications for drug development in malaria.

Prior to her appointment at Rice, Abidi worked as a postdoctoral associate in the Department of Materials Science and Engineering at MIT where she conducted research in Principal Investigator Ming Dao's Nanomechanics Laboratory. Abidi's studies focused on using microfluidics for diagnosis and treatment of red blood cells. Her studies uncovered novel mechanistic insights detailing the adhesion and polymerization processes leading to vaso-occlusive painful crisis in sickle cell disease. Abidi's work also shed light on the critical roles of geometric constraints and cell subpopulation types in the treatment of sickle cell disease.

Abidi's strong record of published work as a postdoctoral researcher at MIT and NY School of Medicine, and as a graduate student at the University of Texas, Austin has been detailed in 10 journal publications. She has three patent applications.

\section{Dr. Renata Fortuna Ramos, Rice University}

Renata Ramos is an Associate Teaching Professor of Bioengineering and the Associate Dean for Academic Affairs of the George R. Brown School of Engineering at Rice University, 6100 Main St., Houston, TX 77005: rfr1@ rice.edu 
WIP: Utilizing Guided Worksheets to Improve Student Performance in Troubleshooting Laboratory Course 


\section{Introduction}

This Work-In-Progress study aims to assess the effect of guided instruction on student learning in commonly misunderstood topics in a troubleshooting lab course. Briefly, the Troubleshooting of Clinically Relevant Devices lab course exposes upper level students to principles of operation, common failures, and preventive maintenance in clinically relevant lab equipment such as the centrifuge, refrigerator, syringe pump, suction pump, microscope and oxygen concentrator. The course is designed to help students learn and practice the troubleshooting process. Students explore basic equipment operating principles, identify failures and repair devices as a team.

In previous course iterations, students expressed unfamiliarity surrounding common parts critical to equipment e.g. solenoid valves, IR sensors (personal experience). Guided inquiry has demonstrated benefits in bridging the scientific gap in knowledge in students as described by Nworgu [1]. To address this unfamiliarity among women and men alike, an intervention in the form of guided worksheets was introduced and its effect on student performance assessed.

\section{Methods}

The course utilizes a flipped classroom format where all students watch lecture videos and complete course readings and pre-labs individually before class. In class, directions for lab procedures and descriptions of failure modes are given to student teams with minimal instruction. At the conclusion of class, students complete a quiz individually assessing technical and troubleshooting knowledge. A team lab report is submitted detailing basic principles, device components, and common failure modes reinforcing the troubleshooting process. Students demonstrate learning through performance on pre-lab, in class performance, quiz and lab report grades.

The guided instruction worksheet intervention required students to identify major subcomponents of key parts in devices often through the form of diagram part identification and brainstorm failure modes of the subcomponents and methods of failure diagnosis.

Worksheets were designed to address topics commonly questioned by students in a low stakes environment. For example, during the centrifuge lab module, students were guided to tear down a DC motor, and to dismantle two different centrifuge models to compare and contrast common points of failure and differences in operation. Students were encouraged to discuss in teams and research as needed.

The control group consisted of students that were encouraged to study and further explore commonly confused topics without any guided instruction worksheets. Equipment such as DC motors and older and newer models of centrifuges were provided for all students to tear down if desired. The additional assignment did not offer any advantage in terms of grade adjustment. The experimental group consisted of students that were instructed to complete the interventional worksheet. Worksheets were evaluated on a completion basis; worksheet performance was not reflected in final grade. The control and experimental activities were added to the course requirements. Because the lab course is offered multiple times a year to senior students, population associated bias was minimized by assigning one lab section as the control section and the other lab section as the experimental section with no direct grade benefit for the designed activities. Due to cost of lab equipment, the lab course usually consists of 12-15 students total. 
For the control group $(\mathrm{n}=12)$, there were 10 males and 2 females. For the experimental group ( $\mathrm{n}$ $=15$ ), there were 3 males and 12 females.

The effect of the guided instruction was assessed using three measures for control and experimental groups: (1) a qualitative survey to evaluate the effect of intervention on knowledge gained in the course using a 5-point Likert scale (2) individual quiz grades and laboratory report team performance, and (3) individual end of semester assessment covering commonly misunderstood topics and application of principles to related topics. Students were made aware that the end of semester quiz was not used in final course grade calculations. The student's $t$ test (two-tailed) was used to determine statistical significance of scores.

\section{Results and Discussion}

Quiz and lab report scores for students did not indicate any statistically significant differences when comparing experimental and control groups (Figure 1, $p>0.01$ ). For the end of course assessment, however, despite the large error bar, a statistically significant difference was observed when comparing experimental and control groups (Figures $1, \mathrm{p}<0.01$, two-tailed ttest). These results suggest that guided instruction increases long-term retention and understanding of the major components and principles of operation of the devices covered in the troubleshooting laboratory modules.

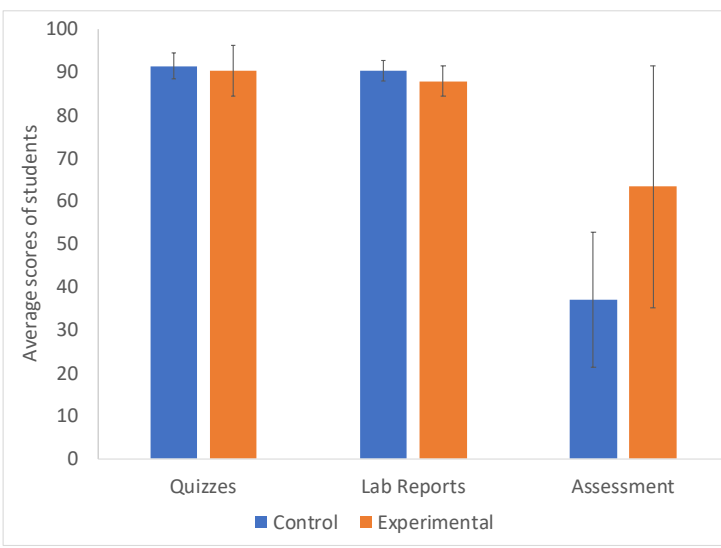

Figure 1. Intervention effect summary using quiz, lab report and end of course assessment scores. Student averages are plotted with error bars representing standard deviation.

The qualitative survey results indicated student perceptions on intervention related activities and their overall confidence in knowledge gained. For the survey statement "Completion of the equipment specific worksheet helped me feel more confident in equipment specific expertise necessary to troubleshoot the associated device", $73 \%$ of the students agreed or strongly agreed on its utility (Figure 2). Overall, majority of students recognized the effect of tear down activities in improving confidence as reflected by survey results (Figure 2).

Because this data is representative of 3 males and 12 females, additional data needs to be collected before any statistically significant difference can be ascertained between genders, if applicable. 
A

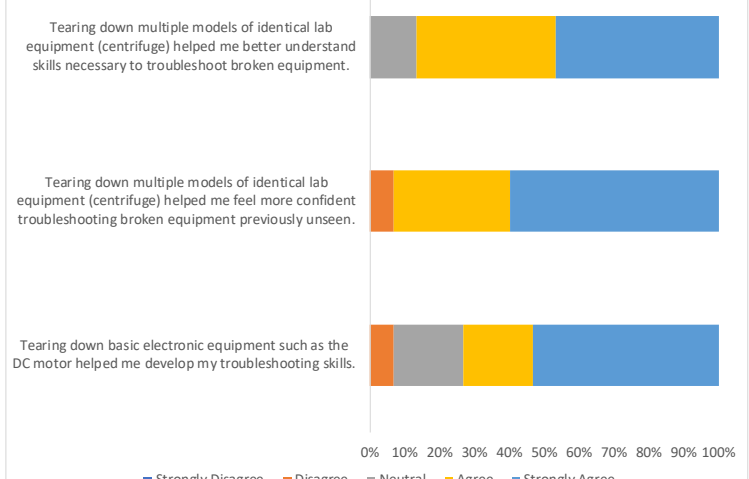

B

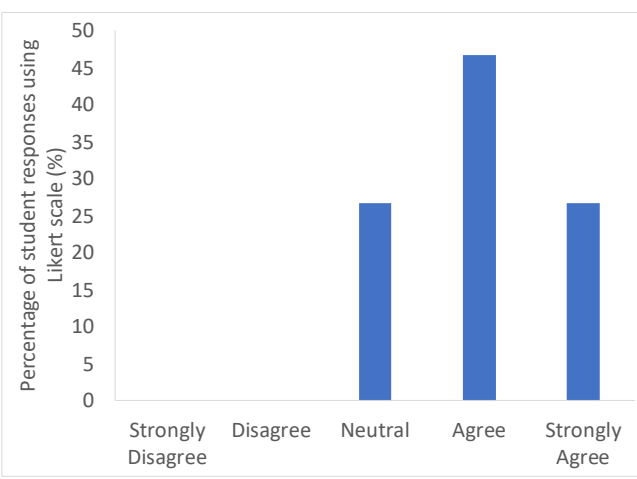

Figure 2. Confidence related effects of intervention. (A) Effect of tearing down equipment on confidence. (B) Worksheet associated effect on confidence. Student responses were measured using a 5-point Likert scale.

Although these results suggest that student learning (based on end of semester assessment) and confidence in troubleshooting skills (as seen in the survey data) increased among students in the experimental group, the effect of the intervention cannot be fully confirmed with such small sample sizes. After the collection of additional control and experimental student data, we hope to report with confidence the effect of our intervention.

\section{References}

1. Nworgu, L.N. and V. V. Otum, "Effect of Guided Inquiry with Analogy Instructional Strategy on Students Acquisition of Science Process Skills," Journal of Education and Practice, 4 (27):35-41, 2013. 